\title{
Comparison of the Fe and Pt Nanoparticles with FePt Alloy Prepared by Polyol Process: Shape and Composition Study
}

\author{
M. FARAHMANDJOU* \\ Department of Physics, Varamin Pishva Branch, Islamic Azad University, Varamin, Iran
}

\begin{abstract}
In this study, Fe and Pt nanoparticles are first synthesized by decomposition of iron(II) chloride tetrahydrate and reduction of platinum(II) acetylacetonate. Then, FePt nanoparticles are similarly fabricated by adding Li$\mathrm{BEt} 3 \mathrm{H}$ to the phenyl ether solution in the presence of oleic acid, oleylamine surfactant at $100{ }^{\circ} \mathrm{C}$, followed by refluxing at $255^{\circ} \mathrm{C}$. The samples were characterized by transmission electron microscopy and energy dispersive spectroscopy analyses after heat treatments. Transmission electron microscopy images show that self-assembled $8 \mathrm{~nm}$ Fe nanoparticles are formed as polygon shape, whereas Pt nanoparticles have broad size distribution. On the other hand, $4.5 \mathrm{~nm}$ FePt nanoparticles have standard division about $9 \%$. The results of energy dispersive spectroscopy analysis reveal that the composition of $\mathrm{Pt}, \mathrm{Fe}$ and FePt nanoparticles gives $\mathrm{Fe}_{56} \mathrm{Pt}_{44}$ stoichiometry.
\end{abstract}

DOI: 10.12693/APhysPolA.123.277

PACS: 81.16.Dn, 81.16.Be, 81.07.-b, 78.67.Bf

\section{Introduction}

In the case of metallic nanoparticles with high magnetic moments like iron oxides, they have to be protected by coating with a surfactant or an inorganic material because due to their large surfaces they are easily oxidized or otherwise subject to corrosion [1]. FePt hard magnetic nanoparticles are of special interest for future ultrahigh-density magnetic recording media [2]. Chemically synthesized magnetic nanoparticles have drawn much attention $[3,4]$ due to the unique magnetic properties derived from small particle sizes and uniform size distribution. Chemical approach is a better way to fulfill this compared with physical ways, since particle size, shape and size distribution can be better controlled in chemical synthesis [5].

Various shapes of nanoparticles have been successfully synthesized over the past years. In particular, dot [5], rod $[6-8]$, spindle $[6,7]$ and tetrapod-shaped $[6,7,9]$ nanoparticles have been fabricated. The properties of nanosized materials are strongly influenced by their shape, it is very important to explore the growth mechanisms that lead to a particular nanocrystal shape to systematically produce the desired material. The solubility of particles of the critical size equals exactly the monomer concentration in the solution [10]. The nucleation stage for the growth of anisotropic shapes plays a key role in determining the size/shape of the resulting nanocrystals. In principle, before the reaction reaches the equilibrium stage, any metastable nanocrystal shapes can be arrested by tuning the reaction conditions [6, 11]. In the present work, the $\mathrm{Fe}$ and $\mathrm{Pt}$ nanoparticles are first fabricated by chemical reduction of $\mathrm{FeCl}_{2} \cdot 4 \mathrm{H}_{2} \mathrm{O}$ and $\mathrm{Pt}(\mathrm{acac})_{2}$ at $250^{\circ} \mathrm{C}$ under $\mathrm{N}_{2}$ atmosphere. Then, $4.5 \mathrm{~nm}$ FePt nanoparticles are fabricated with similar route. The characterization of nanoparticles is studied by scanning electron

*e-mail: farahmandjou@iauvaramin.ac.ir microscopy (SEM) and energy dispersive spectroscopy (EDS) analysis.

\section{Experimental details}

Synthesis of the nanoparticles involves the reduction of $\mathrm{Pt}(\mathrm{acac})_{2}$ and $\mathrm{FeCl}_{2} \cdot 4 \mathrm{H}_{2} \mathrm{O}$ in phenyl ether solvent in the presence of 1,2-hexadecanediol reported by Sun et al. [12]. Oleic acid and oleylamin surfactants were added to the solvent at $100^{\circ} \mathrm{C}$ as a protective agent. The $\mathrm{FePt}$ nanoparticles were formed by adding LiBEt3H superhydride under a blanket of $\mathrm{N}_{2}$ at $195^{\circ} \mathrm{C}$. The refluxing temperature was fixed at $255^{\circ} \mathrm{C}$. The black reaction mixture was cooled to room temperature and then combined with ethanol to remove the impurity. The product was precipitated and separated by centrifugation $(9000 \mathrm{rpm}$, $15 \mathrm{~min}$ ). Any undissolved material was removed by centrifugation machine. To determine the composition of FePt nanoparticles in nucleation and growth stage, EDS analysis $(15 \mathrm{kV})$ was carried out. The specification of the size and shape of nanoparticles was examined by TEM analysis using a Philips EM 208 TEM $(100 \mathrm{kV})$ with a resolution of $200 \mathrm{k} \times$.

\section{Results and discussion}

Figure 1 shows the TEM images of the as-synthesized nanoparticles. In Fig. 1a, the $2-7 \mathrm{~nm} \mathrm{Pt}$ core were created with broad size distribution about $16 \%$ at $190^{\circ} \mathrm{C}$. As one can see, the shapeless $\mathrm{Pt}$ nanoparticles have a broad size distribution. The $8 \mathrm{~nm} \mathrm{Fe}$ nanoparticles as polygon shape were made by similar route with $\mathrm{FeCl}_{2} \cdot 4 \mathrm{H}_{2} \mathrm{O}$ precursor in the same condition. The distance between Fe nanoparticles is close to each other because of magnetic interaction (Fig. 1b).

By adding intense LiBEt3H superhydride to the reaction solution at $195^{\circ} \mathrm{C}$, the $\mathrm{Fe}$ atoms are released from $\mathrm{FeCl}_{2} \cdot 4 \mathrm{H}_{2} \mathrm{O}$ and penetrated to $\mathrm{Pt}$ atoms. In Fig. 1c the 

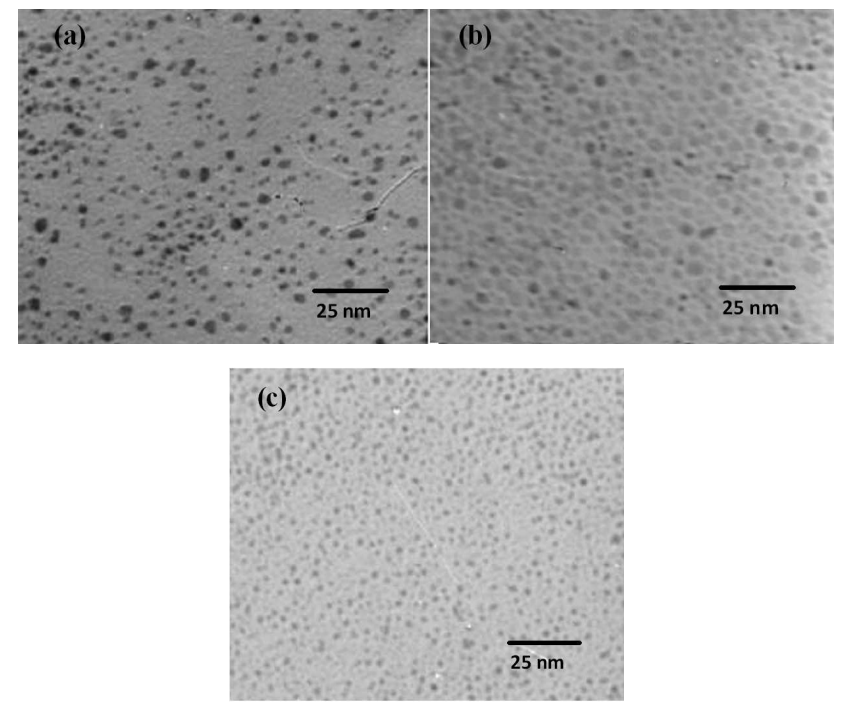

Fig. 1. TEM images of the as-synthesized (a) Pt, (b) Fe, and (c) FePt nanoparticles.

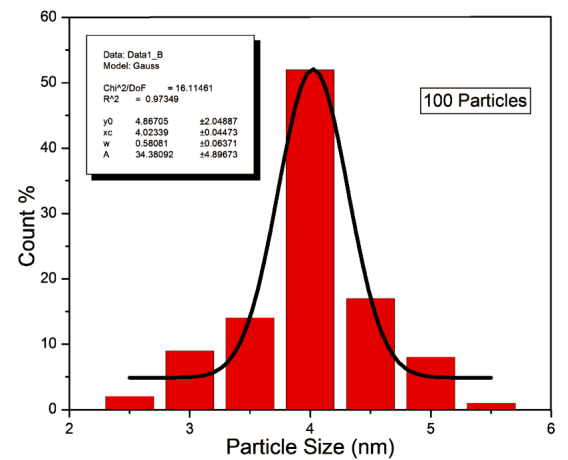

Fig. 2. Particle diameter histogram of FePt nanoparticles.

$4.5 \mathrm{~nm}$ FePt nanoparticles are made by magnetic stability.

Figure 2 shows the size measurement of 100 randomly selected particles fitted with a log normal curve. This figure indicates that self-assembled $4.5 \mathrm{~nm}$ FePt nanoparticles histogram has a narrow size distribution because of magnetic interactions stability with standard deviation about $9 \%$.

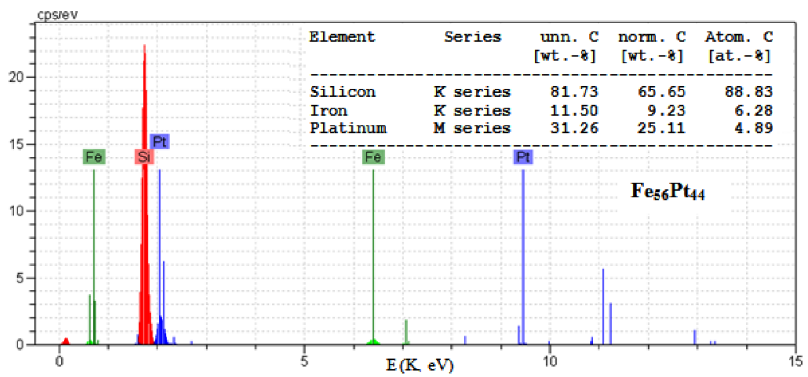

Fig. 3. Energy dispersive spectroscopy (EDS) pattern of nanoparticles.
Figure 3 shows EDS analysis of Fe-Pt nanoparticles. By comparing the area under each peak to a set of standards with known element concentrations, the composition gives $\mathrm{Fe}_{56} \mathrm{Pt}_{44}$ stoichiometry. By adding intense LiBEt3H superhydride at $195^{\circ} \mathrm{C}$ the Fe atoms are released.

\section{Conclusion}

In conclusion, iron-platinum (FePt) nanoparticles have been successfully synthesized with mean diameter of $4.5 \mathrm{~nm}$ by sol-gel method. The TEM results indicate that 2-7 nm Pt nanoparticles have disorder shape. On the other hand, self-assembled $8 \mathrm{~nm} \mathrm{Fe}$ nanoparticles have the regular polygon shape by magnetic interactions. Finally, FePt nanoparticles are fabricated with $3.5 \mathrm{~nm}$ equilibrium distances between particles.

\section{Acknowledgments}

The author is thankful for the financial support of Physics Research Center, Science and Research Campus at Islamic Azad University for analysis and the discussions on the results.

\section{References}

[1] P. Presa, M. Multigner, M.P. Morales, T. Rueda, E. Ferna, A. Hernando, J. Magn. Magn. Mater. 316 $753(2007)$

[2] C.B. Murray, C.R. Kagan, M.G. Bawendi, Ann. Rev. Mater. Sci. 30, 545 (2000)

[3] D. Weller, A. Moser, L. Folks, M.E. Best, W. Lee, M.F. Toney, M. Schwickert, J.U. Thiele, M.F. Dorner, IEEE Trans. Magn. 36, 10 (2000)

[4] S. Sun, C.B. Murray, D. Weller, L. Folks, A. Moser, Science 287, 1989 (2000).

[5] M. Chen, D.E. Nikles, Nano Lett. 2, 211 (2002)

[6] X. Peng, Adv. Mater. 15, 459 (2003)

[7] Z.A. Peng, X. Peng, J. Am. Chem. Soc. 123, 1389 (2001).

[8] S. Link, C. Burda, B. Nikoobakht, M.A. El-Sayed, J. Phys. Chem. B 104, 6152 (2000)

[9] L. Manna, J. Milliron, A. Meisel, C. Scher, A.P. Alivisatos, Nat. Mater. 2, 382 (2003).

[10] C. Burda, X. Chen, R. Narayanan, Chem. Rev. 105, 1025 (2005)

[11] X. Peng, X, J. Wickham, A.P. Alivisatos, J. Am. Chem. Soc. 120, 5343 (1998)

[12] S. Sun, S. Anders, T. Thomson, J.E.E. Baglin, M.F. Toney, H.F. Hamann, C.B. Murray, B.D. Terris, J. Phys. Chem. B 107, 5419 (2003) 